\title{
The effectiveness of acceptance and commitment therapy on perceived stress, resilience, and the quality of life in thalassemia major patients
}

\author{
Fariborz Jabbarifard ${ }^{1}$, Tayebeh Sharifi ${ }^{*}$, Kamal Solati ${ }^{3}$, Ahmad Ghazanfari $^{4}$ \\ ${ }^{1}$ Student Ph.D., Department of Psychology, Shahrekord Branch, Islamic Azad University, Shahrekord, Iran \\ ${ }_{2}^{2}$ Assistant Professor, Department of Psychiatry, Shahrekord Branch, Islamic Azad University, Shahrekord, Iran \\ ${ }^{3}$ Invited Associate Professor, Department of Psychology, Shahrekord Branch, Islamic Azad University, Shahrekord, Iran and \\ Associate Professor, Department of Psychology, School of Medicine, Shahrekord University of Medical Sciences, Shahrekord, \\ Iran \\ ${ }^{4}$ Associate Professor, Department of Department of Psychology, Shahrekord Branch, Islamic Azad University, Shahrekord, Iran
}

*Corresponding Author: Tayebeh Sharifi, Assistant Professor, Department of Psychology, Shahrekord Branch, Islamic Azad University, Shahrekord, Iran. Email: Sharifi_ta@yahoo.com

\begin{abstract}
Background and aims: The present study aimed to determine the effect of acceptance and commitment therapy on perceived stress, resilience, and the quality of life in thalassemia major patients in Lordegan during 2015-2016.

Methods: This semi-experimental study used the pre-test and post-test designs with a control group and period. The samples included 40 patients with thalassemia major who referred to Lordegan Shohada hospital, were selected by the purposeful sampling technique based on the inclusion and exclusion criteria, and were randomly assigned to experimental and control groups. The experimental group received eight-week sessions of the acceptance and commitment-based therapy, while the control group received no intervention until the end of the study. The data were collected by the perceived stress questionnaire (Cohen et al), Researcher Conner and Davison Resilience Scale, and the World Health Organization Quality of Life (SF-26). Finally, the obtained data were analyzed using the SPSS software, repeated measure, and covariance tests

Results: The results showed that there was a significant difference between the experimental and control groups in terms of the perceived stress, resilience, and the quality of life $(P<0.001)$. In addition, based on the results, $66 \%$ of the changes in the perceived stress scores, $81 \%$ of the difference in the resiliency scores, and $75 \%$ of the difference in the quality of life scores were related to the impact of therapy based on the acceptance and group commitment. Further, the results of the analysis variance of the internal group revealed that the effect of therapy based on the acceptance and commitment on the perceived stress, resilience, and the quality of life had a significant difference in pretest, posttest, and follow-up stages.

Conclusion: In general, the therapy based on the acceptance and commitment is found to be a useful practice in reducing the perceived stress while increasing the resilience and improving the quality of life in patients with thalassemia major.

Keywords: Acceptance and commitment therapy, Perceived stress, resilience, Quality of life, Thalassemia major
\end{abstract}

Received: 24 October 2017, Accepted: 25 September 2018, ePublished: 4 April 2019

\section{Introduction}

Thalassemia is a congenital autosomal recessive disease and an inherited hemoglobinopathy result of defection in making the globin chains, that forces the patients to regularly inject blood (for survival) and replace the iron (1). Today, this disorder has changed to a chronic disease $(2,3)$ and is still considered as the most prevalent congenital disorder in the world (4). Iran, with about 20000 thalassemia patients and 3 million thalassemia carriers is one of the countries which are located on the thalassemia border in the world (5). Having a very long and consequent period has affected different aspects of the patients' lifes and led to considerable damage to their health and quality of lifes, as well as to those of their families $(6,7)$. The basic problem occurs when a patient meets the young age and involves in physical problems and functional constraints. Issues such as marriage, higher education, and finding a suitable occupation are among the problems that these patients encounter with in their lives, which increase their level of stress (8). Such stress makes the patients incapable of adjusting with stressful situations which are considered important in reducing one's tolerance to the illness and its constraints, as well as the quality of life. On the other hand, many reasons can cause stress including chronic diseases, therapy costs, and death expectancy, which decrease the quality of life in these patients (6). In this regard, the results of the study by Zeighami Mohammadi et al (8) showed that $72.5 \%$ of

(C) 2019 The Author(s); Published by Shahrekord University of Medical Sciences. This is an open-access article distributed under the terms of the Creative Commons Attribution License (http://creativecommons.org/licenses/by/4.0), which permits unrestricted use, distribution, and reproduction in any medium, provided the original work is properly cited. 
the thalassemia patients reported an average quality of life. In addition, the results of different studies demonstrated that patients with major thalassemia experience further depression and psycho-cognitive psychological disorders while their lower quality of life was compared to that of the healthy ones $(9,10)$. Regarding the above-mentioned cases, the rehabilitation of these patients is of specific importance, which aims at improving the coping ability and promoting the quality of life in these patients. It is a belief in the heat psycho-cognitive field that non-biological factors (i.e., personality-psycho-cognitive factors) contribute to the formation, exacerbation, and success in treating many chronic diseases. Psycho-cognitive therapy is regarded as one of the most effective psycho-cognitive factors. Several studies highlighted the role of psycho-cognitive therapy in the field of health $(11,12)$. In addition, cognitive behavioral therapy is among the psycho-cognitive therapies that can be used to reduce whereas improve the psycho-cognitive problems of patients suffering from thalassemia $(13,14)$. However, nowadays, the third therapeutic generation has been able to obtain the necessary validation to be posed as a replacement for behavioral cognitive therapies that emphasized experimental and basic features in the psycho-cognitive experiences. These therapies seek to increase the relationship between the individual psychocognitive behavior to his feelings and thoughts instead of changing the cognition. In fact, this therapy method helps to achieve a valuable life and more satisfaction through increasing the psycho-cognitive flexibility not just concentrating on cognitive reconstructions. The acceptance- and commitment-based therapy is regarded as one of the most applications of these therapies. This type of therapy includes 6 central processes (i.e., acceptance, causal fault, and the relationship by the present time, self as a base, values, and commitment action) that lead to psycho-cognitive flexibility (15). In therapy which is based on acceptance and commitment attempts to improve the condition of the patients' life by using an example and simile without disturbing their thoughts and feelings in a different way. Various studies conducted in this regard indicated that therapy based on acceptance and commitment has an effect on stress (16), resilience (17), and the quality of life (18). However, there is a lack of research regarding the effect of this therapy on perceived stress, resiliency, and the quality of life in patients with thalassemia major. It seems that negative thoughts and emotions can be treated without being avoided. Further, this therapy can improve these emotions, the tolerance threshold, and ultimately the quality of life in patients with thalassemia.

The current study is important, considering the importance of the perceived stress structures, resilience and the quality of life in these patients. Furthermore, it is necessary due to its chronic and debilitating nature and the positive effects. Reducing these variables can have a role in improving the psycho-cognitive state of these patients. Moreover, given the effectiveness of acceptance and commitment therapy on perceived stress, resilience, and the quality of life in the patients with major thalassemia, the present study sought to answer the question of whether the acceptance and commitment therapy is effective in the perceived stress tolerance and the quality of life of the patients with thalassemia major.

\section{Materials and Methods}

The current semi-experimental study was implemented with pretest-posttest and experimental and control groups with the follow-up design after the approval. Both groups were assessed 3 times per 3 months according to the pretest, posttest, and follow-up protocol. The population of the study included patients with thalassemia major who were within the age range of 18-35 years old and referred to the thalassemia ward in Lordegan hospital during (November) 2016-(July) 2017. A total of 40 patients were selected by purposive sampling method with a confidence coefficient of 0.95 . Moreover, the inclusion criteria included the diagnosis of thalassemia major by oncology specialist, the lack of non-acute or chronic disease other than the major thalassemia, reading literacy, writing and normal IQ, satisfaction with participation in the study, obtaining unsatisfactory scores of perceived stress questionnaires by Cohen et al (19), responding to Conner and Davison (20), and the quality of life of the World Health Organization (SF26). Additionally, the exclusion criteria involved a history of taking psycho-cognitive iatric medications, receiving synchronous psycho-cognitive therapy, being absent more than 2 sessions, failing to perform the assignments. The patients were randomly assigned to 2 experimental and control groups (20 patients in each group). In the present study, the dependent variables before, after, and 3 months after the intervention were measured using 3 questionnaires. Each of the 3 questionnaires is presented in more details as follows.

\section{Perceived Stress Scale}

This scale, introduced by Cohen et al, measures the degree of stressful life situations (19). Perceived Stress Scale Questions are designed in such a way that the respondents express their opinion on the uncontrollable, unpredictable, and suffering from their stress during the past month. These 3 categories are often emphasized as the main components of experiencing stress. In addition, this scale has a number of direct questions about measuring the current levels of the experienced stress in an individual. Further, this instrument has 14 questions, each of which has 5 options. Furthermore, half of the questions are directly scored while the other half are scored in reversely. Moreover, the maximum score is $\mathbf{5 6}$ and the higher score represents more perceived stress. Finally, Sajjadinezhad et al reported a Cronbach $\alpha$ coefficient of 0.75 (21). 
Conner and Davidson resilience scale

The current scale was developed by Connor and Davidson by exanimating the research resources in resilience in order to measure the power of coping with pressure and threats (20). The psycho-cognitive features of this scale were evaluated in 6 groups including the general population, referrals to primary cares, outpatient psycho-cognitive iatric patients, subjects with a generalized disorder, and 2 groups of post-traumatic stress disorder patients. Conner and Davidson declared that this scale could perfectly distinguish between the resilient and non-reflective individuals in clinical and non-clinical groups (20). On this scale, 25 items with a 5-degree Likert-type scale (ranging from totally false to always true) were considered to be scored from zero (completely false) to 4 (always vertebrate) and the questionnaire was given a total score of resilience. Additionally, the validity (through factor analysis, convergent, and divergent validity) and reliability (on the condition of re-test and Cronbach $\alpha$ ) of the scale were established by test developers in normal and atrisk groups (20). Further, the reliability of the scale was reported using Cronbach $\alpha$ of 0.93 In Iran (22).

The quality of life questionnaire of the World Health Organization (Short form)

The quality of life questionnaire of WHO (i.e., a 100-point quality of life scale) was used to measure the quality of life of the patients (23). This questionnaire has 26 items. Two questions are related to satisfaction with general health and general understanding of the quality of life while the remaining questions measure the person's feelings and behavior over the past 2 weeks regarding various aspects of the quality of life such as physical activity, physical pain, and physical role. In other words, a general understanding of the general custodian, vital force, social activity, emotional role, and mental health was measured (23). The scoring of this questionnaire was based on the Likert method on a 5-point gradation and a higher score implied a better quality of life. Questions in 4 general fields included general health, psycho-cognitive aspects, social relationships, and physical environment, along with a record of the positive and negative aspects of the quality of life. Salvation confirmed the validity of this instrument by content validity method (24) with the Cronbach $\alpha$ coefficient of $0.78,0.89,0.95,0.48$, and 0.88 , the public health of 0.78 , and the relationship with close relatives of 0.91. Cronbach's Alpha coefficient of this questionnaire was obtained by Sajjadinezhad et al as 0.81 (21).

After determining the goals of the research at the beginning of therapy, the participants, who were selected based on inclusion and exclusion, were requested to complete the pre-test questionnaires. They were then randomly assigned to experimental and control groups. Subsequently, the participants in the experimental group received acceptance and group therapy for 8 sessions 90 $\mathrm{min} / \mathrm{wk}$ while there was no intervention for the control group during this period. The indicators evaluated in the pre-test were vigorously investigated at the end of the sessions. In addition, a quarterly follow up was conducted. The data were analyzed using descriptive and inferential statistics by SPSS software, version 23. Further, mean and standard deviation, as well as variance analysis with repeated measurements were used as the descriptive and inferential statistics, respectively.

The content of the recognition training sessions and therapy based on acceptance and commitment was adapted from the book of therapy based on the acceptance and commitment of Izadi and Abedi (25), which is presented in Table 1.

\section{Results}

Based on the obtained results, the mean ages in the experimental and control groups were $25.53 \pm 5.06$ and $25.63 \pm 4.18$, respectively. Furthermore, as regards the education level, the patients in the experimental group were under diploma $(15 \%)$, diploma $(60 \%)$, associated

Table 1. The content of therapy sessions based on the acceptance and commitment

\begin{tabular}{|c|c|}
\hline Sessions & Content \\
\hline First & $\begin{array}{l}\text { Performing similarity processes of the group members together, creating a safe and reliable space for the members' comfort, creating a situation } \\
\text { for group solidarity and relating with each other, along with implementing pretest in order to measure dependent variables (i.e., perceived stress, } \\
\text { resilience, and quality of life). }\end{array}$ \\
\hline Second & $\begin{array}{l}\text { Introducing mind and language from the ACT viewpoint, proposing mental products, and presenting the task of the daily experience record of the } \\
\text { mind products. }\end{array}$ \\
\hline Third & $\begin{array}{l}\text { Studying the control strategies, investigating and valuating the control behaviors, as well as avoiding and presenting the consequences of the } \\
\text { control strategies. }\end{array}$ \\
\hline Forth & Training to control the problem, training the internal and external world, and training the acceptance. \\
\hline Fifth & $\begin{array}{l}\text { Specifying values, along with targeting and presenting the value tables to the members, in addition to helping members to recognize their values } \\
\text { and target their values in the presence of the researcher and members. }\end{array}$ \\
\hline Sixth & $\begin{array}{l}\text { Training members about the concepts and faults of frustration, along with training and asking to the members in order to disassociate with their } \\
\text { mental products and address the goals and values by faulting their thoughts. }\end{array}$ \\
\hline Seventh & $\begin{array}{l}\text { Introducing the conceptualized ego of some body organs to themselves, as well as the kinds of ego and learning how it can be as a background by } \\
\text { using values, targeting, and faulting themselves. }\end{array}$ \\
\hline Eighth & $\begin{array}{l}\text { Summing up and preventing recurrence in order to prevent from organs returning to the vain actions. Then, the flexibility psycho-cognitive } \\
\text { hexagonal and the mental pathology hexagonal based on ACT view was taught to the members and finally, the post-test session was performed. }\end{array}$ \\
\hline
\end{tabular}

Abbreviation: ACT, acceptance and commitment therapy. 
degree (5\%), and bachelor (20\%). Moreover, patients in the control group were under diploma $(15 \%)$ or held diploma (55\%), associated (5\%) and bachelor (25\%) degrees. The descriptive statistics of study variables in terms of the group and the type of test are presented in Table 2.

Additionally, as shown in Table 3, analysis of variance (ANOVA) and repeated measure tests are used to examine the effectiveness of the intervention on the perceived stress, resilience, the quality of life. The normality of data is the first presupposition of the test, that is confirmed based on the results of the Kolmogorov-Smirnov test $(P>0.05)$. In addition, the error of variance is another assumption of this analysis. The results of Levine test show that this condition is present in all the variables $(P>0.05)$. Further, the variance-covariance matrix is considered another assumption of this analysis, which is observed based on the results of the Box test $(P>0.05)$. Furthermore, the other assumptions of this test are the Currit assumption, and the results of the test are indicative of non-compliance with this assumption and the use of the Greenhouse correction $(P<0.05)$. Therefore, based on the results of the ANOVA with repeated measurements, the groups are different in the linear composition of the components (Effect size $=0.81$, $P<0.05, \mathrm{~F}=55.33$ ).

The results related to the ANOVA of the intergroup in Table 3 demonstrate a significant difference between the experimental and control groups regarding perceived stress, resilience, and the quality of life $(P<0.001)$. Moreover, the changes can be observed in the perceived stress scores

Table 2. The descriptive statistics of variables in terms of the groups and type of test

\begin{tabular}{llcc}
\hline Subscale & Process & $\begin{array}{c}\text { Experimental } \\
\text { Mean } \pm \text { SD }\end{array}$ & $\begin{array}{c}\text { Control } \\
\text { Mean } \pm \text { SD }\end{array}$ \\
\hline \multirow{2}{*}{ Perceived stress } & Pretest & $45.60 \pm 4.12$ & $44.52 \pm 3.24$ \\
& Posttest & $37.40 \pm 4.05$ & $43.23 \pm 2.61$ \\
& Follow up & $34.40 \pm 4.24$ & $43.61 \pm 3.18$ \\
& Pretest & $24.53 \pm 3.15$ & $25.33 \pm 4.12$ \\
Resilience & Posttest & $41.86 \pm 3.77$ & $25.76 \pm 3.20$ \\
& Follow up & $41.13 \pm 3.83$ & $26.11 \pm 3.43$ \\
Quality of life & Pretest & $66.26 \pm 4.52$ & $67.43 \pm 3.16$ \\
& Posttest & $78.53 \pm 2.09$ & $67.02 \pm 3.76$ \\
& Follow up & $76.32 \pm 2.89$ & $66.32 \pm 2.89$ \\
\hline
\end{tabular}

(66\%), the difference in resiliency scores $(81 \%)$, and the difference in the quality of life scores $(75 \%)$, which are related to the impact of therapy based on the acceptance and group commitment. Additionally, based on the results of the internal group, the effect of therapy based on the acceptance and commitment on perceived stress, resilience, and the quality of life in pretest, posttest, and follow-up stages had a significant difference and the effect is still persistent based on the results of Bonferroni posthoc test and curative therapy during the follow-up period $(P>0.05)$.

\section{Discussion}

The present research was conducted to assess the effect of therapy based on the acceptance commitment on the perceived stress, resilience, and the quality of life of the patients with thalassemia major. On the other hand, therapy based on the acceptance commitment was effective in decreasing the perceived stress in patients with thalassemia major. This effect was persistent during the 3 -month follow-up. This part of findings, directly and indirectly, is in line with the findings of Behrouz et al (26), and Stafford-Brown \& Pakenham (27).

The mechanism of therapy based on the acceptance and commitment in the perceived stress in these patients is argued to be related to a change in the client's attitude at the first session about the irrational and inefficient thoughts regarding the illness and the establishment of the self-created mental constraints concerning the illness, as well as the defection and negative cycle of these thoughts. During the therapy sessions, patients recognized and accepted the constraints of their illness, once more evaluated their capacity, and learned to control their stress through mind-focused exercises. The therapy of acceptance and commitment through the establishment and development of acceptance is believed to increase the practice of values and goals in patients undergoing therapeutic changes and those with thalassemia. In addition, deep discussions about the values and goals of the individual and the necessity of clarifying the values may reduce the severity of the perceived stress. Accordingly, emphasizing on the tendency of individuals to internal experience aims to help them experience their annoying thoughts only as a thought and to be aware of

Table 3. The results of the variance analysis of the subject for differences in components and variance analysis with repeated measurements for differences in pretest, posttest, and follow-up

\begin{tabular}{|c|c|c|c|c|c|c|c|c|c|}
\hline Effect & Process & Source & Total Squares & $d f$ & Squares mean & $F$ & $P$ & Squares & Statistic Potential \\
\hline \multirow{6}{*}{ Join group } & \multirow{2}{*}{ Perceived stress } & Intergroup & 61.40 & 1.43 & 42.67 & 55.33 & 0.001 & 0.66 & 0.87 \\
\hline & & Internal group & 42.20 & 1.43 & 29.32 & 38.03 & 0.001 & 0.57 & 0.85 \\
\hline & \multirow{2}{*}{ Resilience } & Intergroup & 901.06 & 1.27 & 708.95 & 120.42 & 0.001 & 0.81 & 0.98 \\
\hline & & Internal group & 584.08 & 1.27 & 459.55 & 78.06 & 0.001 & 0.73 & 0.92 \\
\hline & \multirow{2}{*}{ Quality of life } & Intergroup & 2646.86 & 1.34 & 1443.72 & 87.29 & 0.001 & 0.75 & 0.92 \\
\hline & & Internal group & 1964.15 & 1.34 & 1463.23 & 64.77 & 0.001 & 0.69 & 0.83 \\
\hline
\end{tabular}


the ineffectual nature of their current program. In fact, individuals should do what is important to them in their lives and in line with their values instead of responding to their thoughts. They can imagine they are the customers who can easily experience the unpleasant internal events at present and separate themselves from the unpleasant reactions, memories, and thoughts. During the therapeutic sessions, patients with thalassemia major were trained to increase their mental and mental acceptance of internal experiences rather than the intellectual and practical avoidance of thoughts and stressful situations. This active and effective encountering with the thoughts and feelings and avoiding of creating a distant, changing the look at oneself, and reevaluating values and goals of life led to success in dealing with life stresses and the perceived stress. Moreover, the results showed the positive effect of therapy based on the acceptance and commitment on increasing the resilience in patients suffering from thalassemia major and its stability after 3 months. This part of the results is directly and indirectly in agreement with the findings of Seyyedjafari et al (17) and Kingston et al (28), which confirmed the role of this therapy in the psycho-cognitive flexibility about the mechanism of the effect of therapy based on the acceptance and commitment on increasing the resilience of patients who suffered from thalassemia major. When the trend of the psycho-cognitive flexibility increased in the process of therapy based on the acceptance and commitment among the patients, they acquired the ability to think flexibly and thus challenged their ineffective and illogical thoughts about their illness. Therefore, they initiated to use their symptoms from replacement justifications and positively rebuild their thought frame. Finally, the patients received more resilience from psychocognitive perspective. As a result, negative events were considered more flexible and realistic, and problems were often considered temporary and limited. During the acceptance and commitment therapy sessions, the mental acceptance of patients about their mental experiences (i.e., thoughts and feelings) increased while ineffective control decreased. The patients were educated to understand that any action to control or avoid the unwanted mental experiences is ineffective and exacerbates their conditions and thus should be experienced without any internal or external reaction removes. Further, education increases the psycho-cognitive awareness of the individual at the instant of the moment, that is, the individual becomes aware of all his mental states, thoughts, and behaviors at each moment and learns to separate himself from these mental experiences (i.e., the separation of knowledge) so that he can be independent of these experiences. Further, the therapy based on the acceptance and commitment leads to a decrease in extreme concentration on selfvisualization of his personal story that an individual has created for himself. These practices assist the individual to display successful flexibility in the problems, as well as the incapable stressful situations and promote their resilience. In general, the central processes of the acceptance based on therapy and commitment to major thalassemia patients explain how to stop or inhibit the worrying thoughts about their illness, constraints, and problems and finally, avoid mixing their annoying, negative, and repetitive thoughts. This leads to more tolerance of the unpleasant excitement and, consequently, resiliency enhancement in patients with major thalassemia. Eventually, it was found that acceptance and commitment therapy improves the quality of life in patients with major thalassemia and this effect is stable even after 3 months. The findings of this section of the present study, directly and indirectly, corroborate with those of several other studies (29-31). Therefore, based on these findings about the effect of the therapy based on acceptance and commitment on the quality of life in patients with thalassemia major, a responsible teaching that involves solving a cognitive-behavioral problem, instantaneous awareness of the emotions (mindfulness), and the unconditional admission of the problem, provides the chances for the patients with major thalassemia to master the skills needed to resolve physical, psychocognitive, and social problems. Because these patients have a lack of cognitive and noncurricular functions, activating these thoughts will only focus on their own self-interest.

Since these patients have multiple cognitive impairments and ineffective thoughts, these thoughts not only help the individual to focus on himself, not to be valuable but to be hopeless to the future, and thus reduce the mood, but also they can be effective in the severity of the signs. Training different challenging methods for illogical thoughts and selecting a correct approach to the problem that has called a cognitive separation in the therapy of commitment and acceptance can have an effect on the quality of life. Moreover, since the aim of the therapy program is to create an attitude or a different relationship with thought, feelings, it can be claimed that the result of the active attention of the patients with thalassemia major to the present time mindfulness improves their quality of life. In conclusion, their adjustment with the disease and their individual problems improve and thus they are able to create more clear aims. In addition, they explain the values and pay attention to specified aims instead of replying to physical signs and everyday issues of their disease. In fact, they accept the thought as thought, feelings as feelings, emotions as emotions, as they are exactly, not more and less that, and this leads to the weaken cognitive frustration. Individuals, along with the acceptance of internal events when they are not in the fight of frustrations and stresses will allow themselves to develop their behavioral sources and commit to a valuable and purposeful life.

\section{Conclusion}

In general, based on the results of this study, treatments based on the acceptance and commitment can be used to 
improve the perceived stress, resilience, and the quality of life in patients with thalassemia major. Further, its effects will be observed in promoting the resilience and the quality of life while reducing the perceived stress. Furthermore, it can reduce the psycho-cognitive problems of this group of patients. By using this intervention, experts can align drug therapies to take effective steps for improving the psycho-cognitive state of thalassemia patients and their families. It is worth mentioning that the results of the present study should be cautiously generalized to other noncommunicable groups and individuals with other diseases.

\section{Conflict of interests}

None.

\section{Ethical considerations}

The study was approved bu the Ethics Committee of Islamic Azad University, Shahrekord Branch (under the ethical code of IR.IAU. SHK.REC.1397.004).

\section{Acknowledgments}

Hereby, we thank the principal and staffs of the Lordegan Shohada hospital who helped us to implement the current research, as well as the participants of the study. This research was part of the Ph.D. thesis which was approved by the Islamic Azad University of Shahre-Kord under the code number of 13320705961007.

\section{References}

1. Al-Harazi AA, Al-Eryani BM, Al-Sharafi BA. Neonatal hemolytic anemia does not always indicate thalassemia: a case report. BMC Res Notes. 2017;10(1):476. doi: 10.1186/ s13104-017-2803-6.

2. Caocci G, Efficace F, Ciotti F, Roncarolo MG, Vacca A, Piras E, et al. Health related quality of life in Middle Eastern children with beta-thalassemia. BMC Blood Disord. 2012;12:6. doi: 10.1186/1471-2326-12-6.

3. Gollo G, Savioli G, Balocco M, Venturino C, Boeri E, Costantini $M$, et al. Changes in the quality of life of people with thalassemia major between 2001 and 2009. Patient Prefer Adherence. 2013;7:231-6. doi: 10.2147/ppa.s42133.

4. Imani E, Asadi Nooghabi F, Hosseini Teshnizi S, Yosefi P, Salari F. Comparison quality of life in patients with thalassemia major based on participating in group activities, Bandar Abbas. The Scientific Journal of Iranian Blood Transfusion Organization. 2013;10(2):198-206.

5. Behrouzian F, Khajehmougahi N, Ziaee Kajbaf A. Relationship of coping mechanism of mothers with mental health of their major thalassemic children. The Scientific Journal of Iranian Blood Transfusion Organization. 2014;10(4):387-93.

6. Kaheni S, Yaghobian M, Sharefzadah GH, Vahidi A, Ghorbani $\mathrm{H}$, Abderahemi A. Quality of life in children with betathalassemia major at center for special diseases. Iran J Ped Hematol Oncol. 2013;3(3):108-13.

7. Haghpanah S, Nasirabadi S, Ghaffarpasand F, Karami R, Mahmoodi M, Parand S, et al. Quality of life among Iranian patients with beta-thalassemia major using the SF-36 questionnaire. Sao Paulo Med J. 2013;131(3):166-72.

8. Zeighami Mohammadi S, Tajvidi M, Ghazizadeh S. The relationship between spiritual well-being with quality of life and mental health of young adults with beta-thalassemia major. The Scientific Journal of Iranian Blood Transfusion Organization. 2014;11(2):147-54.
9. Khani H, Majdi MR, Azad Marzabadi E, Montazeri A, Ghorbani A, Ramezani M. Quality of life of Iranian beta-thalassaemia major patients living on the southern coast of the Caspian Sea. East Mediterr Health J. 2012;18(5):539-45.

10. Ansari S, Baghersalimi A, Azarkeivan A, Nojomi M, Hassanzadeh Rad A. Quality of life in patients with thalassemia major. Iran J Ped Hematol Oncol. 2014;4(2):57-63.

11. Goyal M, Singh S, Sibinga EM, Gould NF, Rowland-Seymour A, Sharma R, et al. Meditation programs for psychological stress and well-being: a systematic review and meta-analysis. JAMA Intern Med. 2014;174(3):357-68. doi: 10.1001/ jamainternmed.2013.13018.

12. Tang YY, Holzel BK, Posner MI. The neuroscience of mindfulness meditation. Nat Rev Neurosci. 2015;16(4):21325. doi: 10.1038/nrn3916.

13. Kahrazei F, Hasemzehi S, Rigi Kooteh B. The Effect of Cognitive Behavioral Therapy on Components of Anxiety (Physical, Behavioral and Cognitive) in Patients with Thalassemia Major. Middle Eastern Journal of Disability Studies. 2017;7:49.

14. Kiani J, Pakizeh A, Ostovar A, Namazi S. Effectiveness of cognitive behavioral group therapy (CBGT) in increasing the self esteem \& decreasing the hopelessness of beta-thalassemic adolescents. Iran South Med J. 2010;13(4):241-52.

15. Hayes SC, Luoma JB, Bond FW, Masuda A, Lillis J. Acceptance and commitment therapy: model, processes and outcomes. Behav Res Ther. 2006;44(1):1-25. doi: 10.1016/j. brat.2005.06.006.

16. Sabour S, Kakabraee K. The Effectiveness of Acceptance and Commitment Therapy on Depression, Stress and Indicators of Pain in Women with Chronic Pain. Iranian Journal of Rehabilitation Research in Nursing. 2016;2(4):1-9. doi: 10.21859/ijrn-02041.

17. Seyyedjafari J, Motamedi A, Mehradsadr M, Olamaie Kopaei M, Hashemian S. The Effectiveness of Acceptance and Commitment Therapy (ACT) on Resilience in Elderlies. Journal of Aging Psychology. 2017;3(1):21-9.

18. Ghomian S, Shairi MR. The effectiveness of acceptance and commitment therapy for children with chronic pain on the quality of life on 7 to 12 year-old children. Int J Pediatr. 2014;2(2,3):47-55.

19. Cohen S, Kamarck T, Mermelstein R. A global measure of perceived stress. J Health Soc Behav. 1983;24(4):385-96.

20. Connor KM, Davidson JR. Development of a new resilience scale: the Connor-Davidson Resilience Scale (CD-RISC). Depress Anxiety. 2003;18(2):76-82. doi: 10.1002/da.10113.

21. Sajjadinezhad MS, Asgari K, Molavi H, Adibi P. Comparing the Effectiveness of Cognitive-Behavioral Stress Management, Optimism Training and Medical Therapy on Somatic Symptoms, Perceived Stress, Illness Perception and Quality of Life in Patients with Ulcerative Colitis. Journal of Arak University of Medical Sciences. 2016;18(103):40-54.

22. Jowkar B. The mediating role of resilience in the relationship between general and emotional intelligence and life satisfaction. Contemporary Psychology. 200;2(4):3-12.

23. World Health Organization (WHO). WHOQOL: Measuring Quality of Life. Geneva: WHO; 1997. Available from: https:// www.who.int/mental_health/media/68.pdf.

24. Nejat S. Quality of life and its measurement. Iran J Epidemiol. 2008;4(2):57-62

25. Izadi R, Abedi M. Therapy based on acceptance and commitment. Tehran: Forest Pub; 2014. [Persian].

26. Behrouz B, Bavali F, Heidarizadeh N, Farhadi M. The effectiveness of acceptance and commitment therapy on psychological symptoms, coping styles, and quality of life in patients with type-2 diabetes. Journal of Health. 
2016;7(2):236-253. [Persian].

27. Stafford-Brown J, Pakenham KI. The effectiveness of an ACT informed intervention for managing stress and improving therapist qualities in clinical psychology trainees. J Clin Psychol. 2012;68(6):592-13. doi: 10.1002/jclp.21844.

28. Kingston T, Dooley B, Bates A, Lawlor E, Malone K. Mindfulness-based cognitive therapy for residual depressive symptoms. Psychol Psychother. 2007;80(Pt 2):193-203. doi: 10.1348/147608306x116016.

29. Nasiri S, Ghorbani M, Adibi P. The effectiveness of acceptance and commitment therapy in quality of life in patients with functional gastro intestinal disorder. J Clin Psychol. 2016;7(4):93-102.

30. Luciano JV, Guallar JA, Aguado J, Lopez-Del-Hoyo Y, Olivan B, Magallon R, et al. Effectiveness of group acceptance and commitment therapy for fibromyalgia: a 6-month randomized controlled trial (EFFIGACT study). Pain. 2014;155(4):693-702. doi: 10.1016/j.pain.2013.12.029.

31. Wicksell RK, Kemani M, Jensen K, Kosek E, Kadetoff D, Sorjonen $K$, et al. Acceptance and commitment therapy for fibromyalgia: a randomized controlled trial. Eur J Pain. 2013;17(4):599-611. doi:10.1002/j.1532-2149.2012.00224.x. 